\title{
Operational Readiness of Traffic Signal System with Human Error under Environmental Conditions Using Neural Network Approach
}

\author{
Neelam Sharma ${ }^{1}$, Surbhi Gupta ${ }^{2}$, Ekata $^{3}$ \\ ${ }^{I}$ Applied Sciences Department Krishna Institute of Engineering and Technology, Ghaziabad, India \\ ${ }^{2}$ Mathematics Department AMITY University Noida, India \\ ${ }^{3}$ Senior IEEE Member Applied Sciences Department Krishna Institute of Engineering and Technology, \\ Ghaziabad, India
}

\begin{abstract}
Recent approach of Neural Network can be employed to solve a wide spectrum of problem in optimization. This paper deals with reliability, non-reliability and cost analysis of a traffic signal system. As all knows, traffic system consists of three lights; red, green and yellow. The red and green signal lights pre-empt in repair over yellow signal light. A multilayered neural network model is introduced in order to optimize the maintenance of the traffic signal system. The system can be fail due to hardware failure with human errors and various environmental conditions. All types of failures, repairs and waiting rates are exponential. System state probabilities and other parameters are developed for the proposed model using neural network approach. At the last, numerical examples are included to illustrate the results.
\end{abstract}

Keywords: Neural Network, reliability, non-reliability, Neural weights, cost factor.

\section{Introduction}

Complexity of system(s) escalates the degradation of functioning of system. The enforcement of system/ components confide on the judgment taken during designing, implementing, operating and maintaining. Reliability analysis advices identification of the technical circumstances and predicts the system life in future. Also cost factor, significantly, reveals the economical conditions that help in decision making. The cost and reliability of multi-component system have been studied by many earlier researchers [3,4,5,7]. Current developments in advanced traffic control techniques are giving rise to an increasing requirement for reliable near-future forecasts of traffic flow. Control of traffic signal for efficient movement of traffic on urban streets constitutes a challenging part of an urban traffic control system. Traffic signal control varies in complexity from simple systems that use historical data to determine fixed timing plans, to adaptive signal control, which optimize timing plans for a network of signals accordingly to traffic condition in real time. The traffic flow behavior in the network depends on control inputs that are directly related to corresponding control devices, such as traffic lights.

Most of the time classical mathematical approaches are too involve solving the mathematical equations of complex system. Soft computing methods (such as Neural Network, fuzzy logic, probabilistic reasoning etc.) have been employed to tackle such type of complex problems [1,2]. Neural network can be employed to solve extended problems in optimization, parallel computing, matrix algebra and signal processing. A study on the applicability of different kind of neural networks for the probabilistic analysis is prominent now-a-days. Various researchers have been performing hardware implementations in diverse pattern [8, 9, 11, 12,15].

Proposed neural network design based on models of the biological neuron system [7]. Neural networks are also recognized by their learning mechanism i.e. it consists of three components viz., neurons, network architecture and learning algorithm. It consists of number of layers that can be divided into three main parts [Fig.1]. The first part, which communicates with environment, is known as input layer. The architecture of this class also has one or more intermediary layers named as hidden layers. The third and last part presents the results to the user, named output layer. Different layers are connected through synaptic links carrying the weights [Fig. 2] [8, 10]. Learning algorithm describes the process to adjust weights that minimize the errors of the network outputs. These networks can learn automatically, complex relationships among data. Thus, this technique is very useful in modeling processes for which mathematical modeling is difficult.

Keeping these facts in mind, authors established and solved mathematical model for traffic signal system using neural networks. The main objective of this paper is to focus the new encoding method to predict the cost and reliability of traffic signal system using neural network approach. 


\section{System Description}

Real-time traffic signal control is an integral part of the urban traffic system [13,14]. Traffic signals adequate for orderly movement of traffic and avoid traffic delays, fuel consumption, accidents and discourtesy of traffic rules in legitimate but may be inadequate when installed improperly.

The system considered based on three colour signals, namely, red, green and yellow. On failure of yellow colour signal, the system works in reduced efficiency state. The red and green signal lights pre-empt in repair over yellow signal light. Pre-emptive repeat policy has been adopted for repair purpose. The whole system can also fail due to environmental conditions such as defective roadways, poorly placed signals or inclement weather etc. and human error such as high speed driving, not follow the rules of traffic, distraction of mind, overtaking etc. $[6,16,17]$. The system has to wait for repair in case of environmental failure.

Fig. 2 shows the transition diagram of traffic signal system, which classifies the whole working of the system. Neural network needs information for its output to train the network for new input. In practical life, a priori knowledge of accurate estimation of reliability parameters is beneficial in decision making. The principle causes of unreliability are design deficiencies, unsuspected material incompabilities, lack of capability, unavoidable complexities. In the field of reliability failure plays a vital role. All failure and repair rates are predicted in some fixed time interval and established as neural weights. Many of the algorithms used to train neural networks [1] but here Back propagation is conducted for determining the weights of the neural network. The complete process of determining and adjusting the weights is repeated whenever the desired reliability not obtained. MATLAB program has been developed to solve model generated. The Feed forward algorithm is used in the programming. Simulation results reveal significant improvement in traffic control using neural network approach.

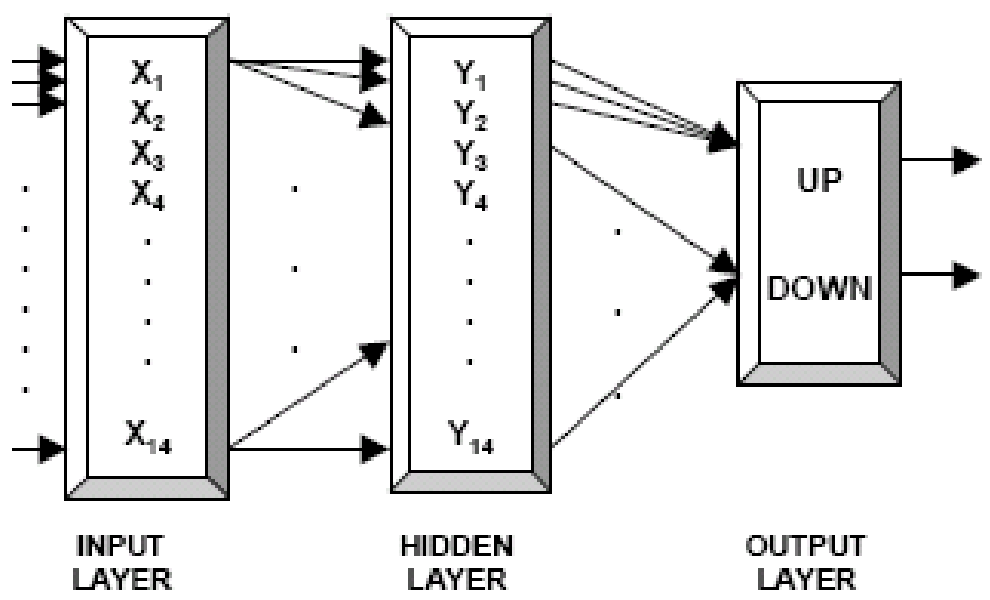

Fig. 1: Neural network structure

Following are the various states of Traffic signal system:

$\mathrm{P}_{\mathrm{o}}$ : $\quad$ All units are in operable state.

$\mathrm{P}_{\mathrm{R}}$ : $\quad$ System completely fails due to failure of Red signal unit

$\mathrm{P}_{\mathrm{G}}$ : $\quad$ System completely fails due to failure of Green signal unit

$\mathrm{P}_{\mathrm{Y}}: \quad$ System works with lesser efficiency when Yellow signal unit fails

$\mathrm{P}_{\mathrm{YR}}: \quad$ System fails from degraded state due to failure of Red signal unit

$\mathrm{P}_{\mathrm{YG}}$ : $\quad$ System fails from degraded state due to failure of Green signal unit

$\mathrm{P}_{\mathrm{YH} 2}$ : System fails from degraded state due to Human error

$\mathrm{P}_{\mathrm{E}}{ }_{\mathrm{W}}$ : $\quad$ System fails due to Environmental conditions and waiting for repair

$\mathrm{P}_{\mathrm{E}}{ }^{\mathrm{R}}: \quad$ System fails due to Environmental conditions and ready for repair

$\mathrm{P}_{\mathrm{H} 1}$ : System fails due to Human error 


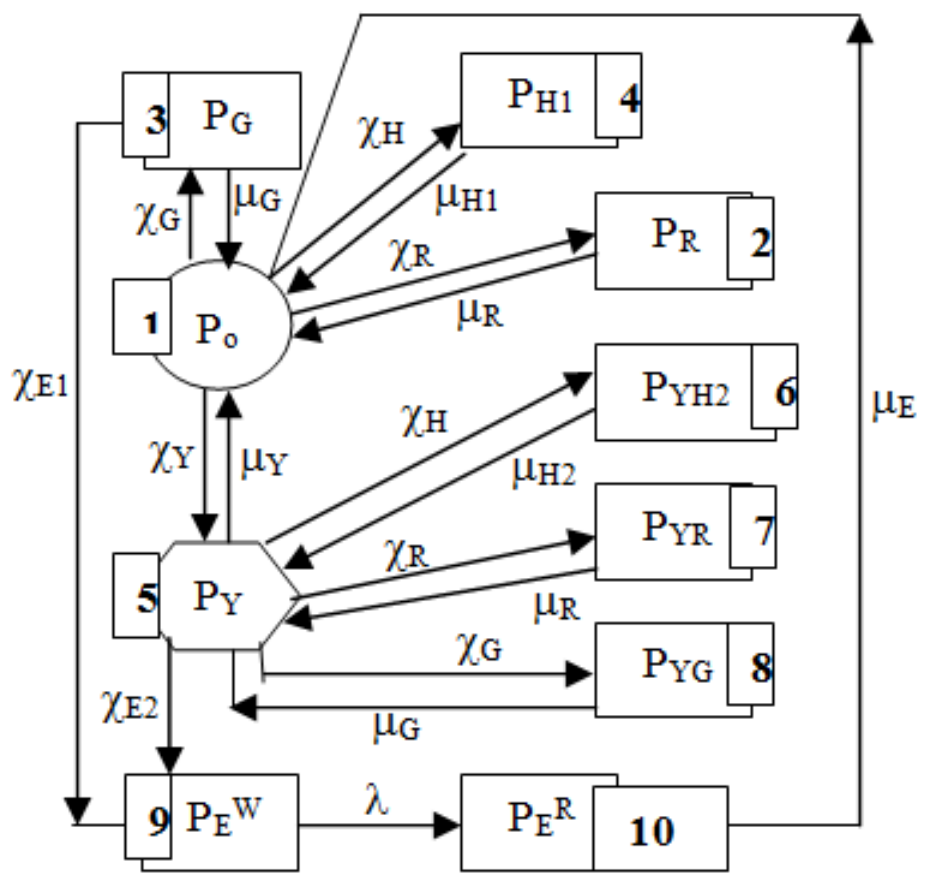

Fig. 2: Functional Block Diagram

1. Initially all the units are fully functional.

\section{Assumptions}

2. The system works with lesser efficiency due to the failure of Yellow signal.

3. The system fails completely due the failure of Red and Green signal.

4. The red and green signal lights pre-empt in repair over yellow signal light.

5. All the failure and repair rates of the system are statistically independent.

6. The failure and repair rates of the system are established as neural weights.

7. The residual subsystem can't fail from the failed state.

8. Repaired subsystem(s) work(s) like new.

\section{Algorithm of Feed forward Neural Network of The System}

Step 1 : Assume number of inputs and outputs

Step 2 : Normalize the inputs and outputs.

Step 3 : Assume the number of neuron in hidden layer.

Step 4 : Initialize the weight matrices

[W]: Weights of synapses connecting input and hidden layer.

[V]: Weights of synapses connecting hidden and output layer.

Step 5 : Compute inputs of hidden layer

$\mathrm{Ih}=\mathrm{W}^{\mathrm{T}} * \mathrm{Oi}$

Step 6 : Evaluate output of hidden layer using sigmoidal function

Step 7 : Compute inputs of output layer

$\mathrm{Io}=\mathrm{V}^{\mathrm{T}} * \mathrm{Oh}$

Step 8 : Evaluate output of output layer using sigmoidal function

Step 9 : Calculate the error

Step 10 : If error < tolerance

then End learning process.

else if epochs < limit

then Update weight matrices [V] and [W]

epochs incremented by 1

go to Step 5

else End learning process.

A. Notations

$P_{i}(t): \quad \quad \quad \quad \quad \quad$ Probability of $i^{t h}$ state at any time $t$.

$P_{i}(t+\Delta t): \quad$ Probability of $i^{\text {th }}$ state at time $(t+\Delta t)$. 


\begin{tabular}{|c|c|}
\hline$\chi_{G}$ & $\begin{array}{l}\text { Failure rate due to failure of Green } \\
\text { signal. }\end{array}$ \\
\hline$\chi_{R}:$ & $\begin{array}{l}\text { Failure rate due to failure of Red } \\
\text { signal. }\end{array}$ \\
\hline$\chi_{Y}:$ & $\begin{array}{l}\text { Failure rate due to failure of Yellow } \\
\text { signal. }\end{array}$ \\
\hline$\chi_{H 1}:$ & Failure rate due to Human error. \\
\hline$\chi_{H 2}:$ & $\begin{array}{l}\text { Failure rate due to Human error when } \\
\text { system works with lesser efficiency. }\end{array}$ \\
\hline$\chi_{E I}:$ & $\begin{array}{l}\text { Failure rate due to Environmental } \\
\text { conditions. }\end{array}$ \\
\hline$\chi_{E 2}:$ & $\begin{array}{l}\text { Failure rate due to Environmental } \\
\text { conditions when system works with } \\
\text { lesser efficiency. }\end{array}$ \\
\hline$\mu_{\mathrm{G}}:$ & $\begin{array}{l}\text { Repair rate due to repair of Green } \\
\text { signal. }\end{array}$ \\
\hline$\mu_{\mathrm{Y}}:$ & $\begin{array}{l}\text { Repair rate due to repair of Yellow } \\
\text { signal. }\end{array}$ \\
\hline$\mu_{\mathrm{R}}:$ & $\begin{array}{l}\text { Repair rate due to repair of Red } \\
\text { signal. }\end{array}$ \\
\hline$\mu_{\mathrm{H} 1}$ & Repair rate of Human error. \\
\hline$\mu_{\mathrm{H} 2}$ & $\begin{array}{l}\text { Repair rate of Human error when } \\
\text { system works with lesser efficiency. }\end{array}$ \\
\hline$\mu_{\mathrm{E}}$ & $\begin{array}{l}\text { Repair rate of Environmental } \\
\text { conditions. }\end{array}$ \\
\hline$\lambda:$ & Waiting repair time \\
\hline
\end{tabular}

\section{B. Neural Network of the System}

Fig. 1 represents the neural network of the system. The network consists of three layers viz., an input layer, an output layer and a hidden layer. The number of neurons in input and hidden layer are equal to number of states in transition diagram, usually determined on basis of observations. Each state has a rule of working as mentioned above. The failure and repair rates are established as neural weights. Numbers of neurons in output layer represent the reliability and unreliability of the system.

At any time t during operation of the system as represents in Fig. 2, the inputs are as follows:

$X_{i}=P_{i}(t) ;$ where $i=1,2,3, \ldots, 9$

The weights of the neural network related to the system model are

$$
\begin{aligned}
& \omega_{12}=\chi_{R} \Delta t=\omega_{57} \\
& \omega_{13}=\chi_{G} \Delta t=\omega_{58} \\
& \omega_{14}=\chi_{H 1} \Delta t \\
& \omega_{15}=\chi_{Y} \Delta t \\
& \omega_{19}=\chi_{E 1} \Delta t \\
& \omega_{11}=1-\omega_{12}-\omega_{13}-\omega_{14}-\omega_{15}-\omega_{19} \\
& \omega_{21}=\mu_{R} \Delta t=\omega_{75} \\
& \omega_{22}=1-\omega_{21} \\
& \omega_{31}=\mu_{G} \Delta t=\omega_{85} \\
& \omega_{33}=1-\omega_{31} \\
& \omega_{41}=\mu_{H 1} \Delta t \\
& \omega_{44}=1-\omega_{41}
\end{aligned}
$$




$$
\begin{aligned}
& \omega_{51}=\mu_{Y} \Delta t \\
& \omega_{56}=\chi_{H 2} \Delta t \\
& \omega_{59}=\chi_{E 2} \Delta t \\
& \omega_{55}=1-\omega_{56}-\omega_{57}-\omega_{58}-\omega_{59}-\omega_{51} \\
& \omega_{65}=\mu_{H 2} \Delta t \\
& \omega_{66}=1-\omega_{65} \\
& \omega_{77}=1-\omega_{75} \\
& \omega_{88}=1-\omega_{85} \\
& \omega_{9(10)}=\lambda \Delta t \\
& \omega_{99}=1-\omega_{9(10)} \\
& \omega_{(10) 1}=\mu_{E} \Delta t
\end{aligned}
$$

\section{Equations}

The basic equations of neural network are represented in the following form

$$
\begin{aligned}
Y_{i}= & P_{i}(t+\Delta t) \text { where } i=1,2, \ldots, 9 \\
Y_{1} & =\omega_{11} X_{1}+\omega_{21} X_{2}+\omega_{31} X_{3}+\omega_{41} X_{4} \\
& +\omega_{51} X_{5}+\omega_{(10)} X_{(10)} \\
Y_{2} & =\omega_{12} X_{1}+\omega_{22} X_{2} \\
Y_{3} & =\omega_{13} X_{1}+\omega_{33} X_{3} \\
Y_{4} & =\omega_{14} X_{1}+\omega_{44} X_{4} \\
Y_{5} & =\omega_{15} X_{1}+\omega_{55} X_{5}+\omega_{65} X_{6}+\omega_{75} X \\
& +\omega_{85} X_{8} \\
Y_{6} & =\omega_{56} X_{5}+\omega_{66} X_{6} \\
Y_{7} & =\omega_{57} X_{5}+\omega_{77} X_{8} \\
Y_{8} & =\omega_{58} X_{5}+\omega_{88} X_{8} \\
Y_{9} & =\omega_{59} X_{5}+\omega_{19} X_{1}+\omega_{99} X_{9} \\
Y_{(10)} & =\omega_{(10)(10)} X_{(10)}+\omega_{9(10)} X_{9}
\end{aligned}
$$

\section{Experimental Results}

The proposed approach has been tested on the data from traffic signal system. Fig. 3(a) shows the desired reliability of the system with increasing number of iterations. Of course, better results can be obtained if more iteration is performed. Fig. 3(b) shows the unreliability of the system with iterations. The results exhibit the affected reliability with diversifies time.

In addition, cost analysis with time is evaluated by following mathematical expression

$$
G(t)=C_{1} * P_{u p}(t)-C_{2}(t)-C_{3}
$$

where $\mathrm{C} 1$ : revenue cost

$\mathrm{C} 2$ : repair cost per unit time

C3: system establishment cost

$P_{u p}(t)$ : Probability of operable states 
Fig. 4 depicts the distinct profit graphs for different values of revenue and repair cost, under one time establishment cost.

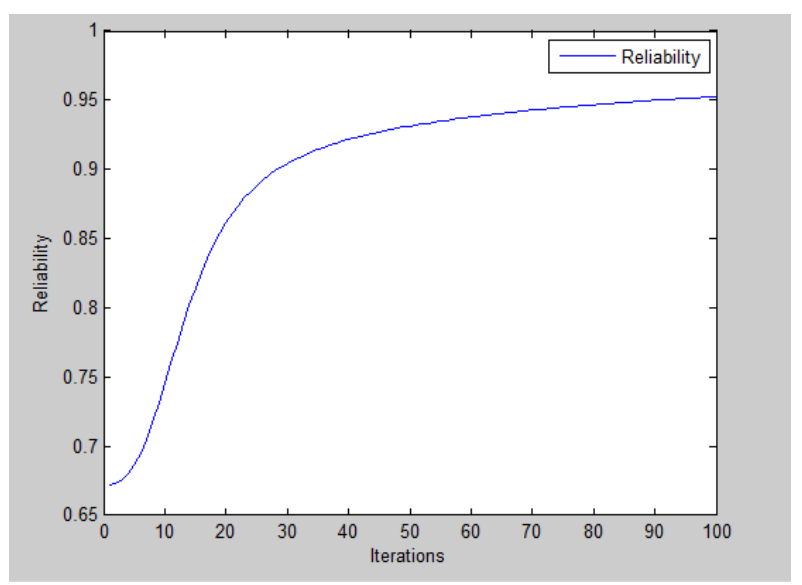

Fig. 3(a): Time vs Reliability

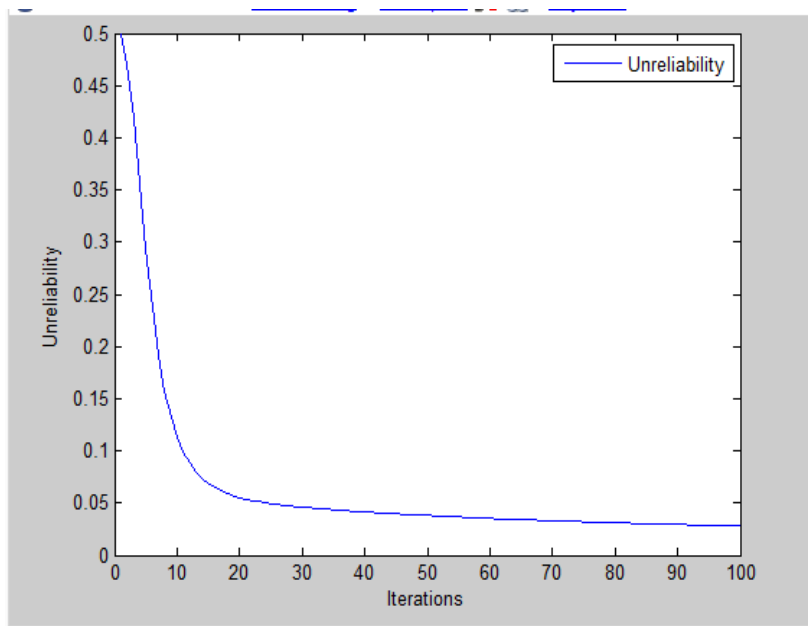

Fig. 3(b): Time vs Unreliability

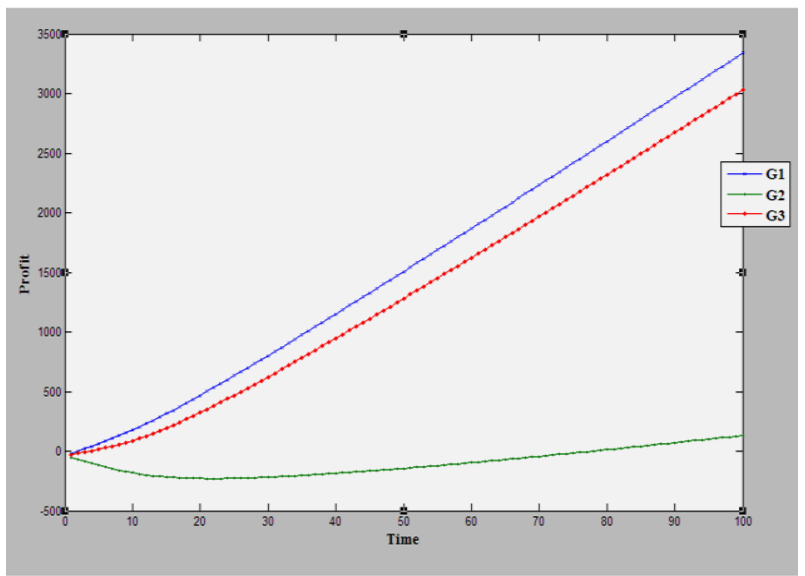

Fig. 4: Time vs Comparative cost

VI. VConclusions

In this paper, the neural network approach for analytical study of reliability of traffic signal system is discussed. Comparative study of the reliability with time is presented. Fig. 3(a) and Fig. 3(b) exhibit the application of neural network approach in reliability measures. Fig. 4 shows the profit of the system in long time which will help to the economical analysts. 
Neural networks in various reliability factors can achieve good performance. The field of neural hardware implementation is undoubtedly very vast and completely open for research till this moment. Our contribution is merely a step forward and an effort to explore such important technological field with viable implementation technique. It is hoped that this work will serve as a valuable resource for real time applications.

\section{References}

[1]. N. Karunanithi, D. Whitley, Y. K. Malaiya, 'Using neural networks in reliability', IEEE, Vol. 9, pp 53-59, Jul 1992.

[2]. Ikuo Yamamoto, 'Application of neural network to marine vehicle', IEEE, pp 220-224, 1995.

[3]. L. Britler Alan, Crystal D. Sloan, 'System reliability Prediction: Towards a general approach using neural network', Nashville, Tennessee, American Institute of Aeronautics and Astronautics, 2005.

[4]. Ronald E. Giutini, 'Mathematical characterization of human reliability for multitask system operations', IEEE, pp 1325-39, 2000.

[5]. Igor Bazovsky, Reliability theory and practice, PHI Englewood cliff, NJ, 1961.

[6]. Mallikarjuna Reddy, K.L.N. Rao, 'Neural network for the reliability analysis of a series-parallel system subjected to common-cause and human error failures', Bulletin of pure and applied Sciences, vol.21E(no.1), pp 251-257, 2002.

[7]. Rajasekaran, S., Pai, G.A. Vijaylakshmi, Neural Networks, Fuzzy Logic, and Genetic Algorithms, PHI, 2004.

[8]. S. Lolas, O. A. Olatunbosun, "Prediction of vehicle reliability performance using artificial neural networks", Elsevier, Science direct, Expert systems with applications 34, pp 2360-2369, 2008

[9]. K.W. Chau, "Reliability and performance-based design by artificial neural network", Elsevier, Science direct, Advances in Engineering Software 38, pp 145-149, 2007.

[10]. Su, Yu-shen, Huang, Chin Yu, “ Neural - network- based approaches for software reliability estimation using dynamic weighted combinational models", Elsevier, the journal of systems are Software 80, pp 606-6015, 2007.

[11]. Ekata, Neeraj Gupta and S.B. Singh, "Operational availability of marine vehicle system using neural network approach", IJCSM, vol. 2,pp. 91-99, 2010.

[12]. Ekata, Neelam Sharma, C.M. Batra, "Neural Network Approach for Analytical Study of the Reliability of Refrigeration System", IEEE International Conference on Innovative Applications of Computational Intelligence on Power, Energy and Controls with their impact on Humanity (CIPECH-14) at Krishna Institute of Engineering and Technology, Ghaziabad on 28 - 29 November 2014 pp. 511-514.

[13]. Dipti srinivasan, Min Chee Choy and Ruey Long Cheu "Neural Network For Real-Time Traffic Signal Control” IEEE VOL.7 ,NO 3, September 2006.

[14]. L.Zang, L.Jia, and Y. Luo, “An Intelligent Control Method for Urban Traffic Signal Based on Fuzzy Neural Network," in Proc.6 ${ }^{\text {th }}$ World Congress on Intelligent Control and Automation, 2006, Dalian, China.

[15]. Ekata, Praveen Kumar Tyagi, Harshit singhal "Neurofuzzy Inference System for Diagnosis of Leukemia" IEEE International Conference on Innovative Applications of Computational Intelligence on Power, Energy and Controls with their impact on Humanity (CIPECH-14) at Krishna Institute of Engineering and Technology, Ghaziabad on 28- 29 November 2014, pp 461-465.

[16]. https://transportation.arlingtonva.us/streets/traffic-signals/

[17]. http://www.jhpolice.gov.in/road-safety/common-causes-of-road-accidents

Dr. Neelam Sharma (M.sc, M.Phil, and Ph.D) is a Associate Professor in the field of Mathematics in Krishna

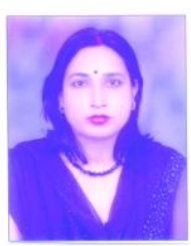
Institute of Engineering and Technology Ghaziabad. She has Sixteen years experience of teaching B.Sc. and B.Tech. Students. She has been published more than 10 research papers in various national and international Journals. She has published two books related to Engineering topics. She has attended many International Conferences and O.I.P for the purpose to enhance the research work and Knowledge. Her research interest includes Soft Computing and Reliability Engineering

Dr. Surbhi Gupta (M.sc.,M.phil. P.hd.) She is a Assistant Professor in the field of Mathematics in Amity

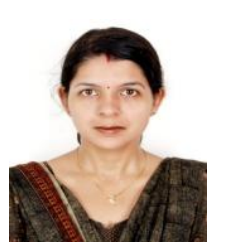
University Noida. She has twelve years experience of teaching B.tech and M.tech students. She have published Research Papers in various International Journals and also guided the P.hD. students for their research project.

Dr. Ekata is a Associate Professor in the Department of Applied Sciences, Krishna Institute of Engineering and

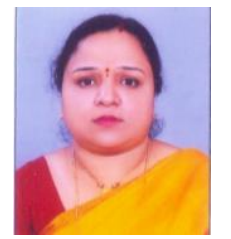
Technology, Ghaziabad. She has around 18 years of teaching and research experience. She has received versatile degrees Ph.D. in Mathematics and Master in Computer Sciences. She is a senior member of IEEE-Computational Intelligence Society. She is a regular reviewer of International Journals. He has been a member of organizing committee of workshops and international conferences. She has coauthored of two books on different courses of Applied Mathematics. She has been conferred with one of best teacher award and received many Certificates of Appreciation for superior performance in her college. She has published several research papers in reputed International and National Journals. Her area of research is Reliability Theory and also work on Neural Network, Fuzzy Logic, Genetic Algorithm. 